International Journal of Pure and Applied Mathematics

Volume 108 No. 4 2016, 767-780

ISSN: 1311-8080 (printed version); ISSN: 1314-3395 (on-line version)

url: http://www.ijpam.eu

doi: 10.12732 /ijpam.v108i4.3

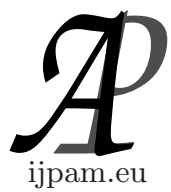

\title{
RECURRENCE RELATIONS AND SEMILOCAL CONVERGENCE OF A FIFTH ORDER METHOD IN BANACH SPACES
}

\author{
J.P. Jaiswal ${ }^{1} \S$, Bhavna Panday ${ }^{2}$ \\ ${ }^{1}$ Department of Mathematics \\ Maulana Azad National Institute of Technology \\ Bhopal, M.P., 462051, INDIA \\ ${ }^{2}$ Department of Mathematics \\ DMS Regional Institute of Education \\ Bhopal, M.P., 462013, INDIA
}

\begin{abstract}
The aim of this article is to study the semilocal convergence of a fifth order method in Banach spaces. Using recurrence relations, we have proved convergence, existence and uniqueness theorem, along with a priori error bounds which shows the $\mathrm{R}$-order of convergence. Finally, we have demonstrated the numerical results on nonlinear integral equation.
\end{abstract}

AMS Subject Classification: 65D10, 65D99

Key Words: nonlinear equation, error bound, Banach space, recurrence relation, semilocal convergence

\section{Introduction}

The basic problem in applied mathematics is finding the solution of nonlinear equations as follows

$$
T(x)=0,
$$

where $T: \Omega \subseteq X \rightarrow Y$ is a nonlinear operator on an open convex subset $\Omega$ of a Banach space $X$ with values in a Banach space $Y$. Newton's method

Received: March 28, 2016

Published: August 16, 2016

$\S$ Correspondence author
(C) 2016 Academic Publications, Ltd.

url: www.acadpubl.eu 
is a well known scheme for solving nonlinear equations, which has second order convergence. In [1], Kantorovich established semi local convergence of the Newton's method. Rall [2], has proposed a different approach for the semilocal convergence of the same scheme, which based on recurrence relations. Nowdays, innumerable variant of Newton's method with higher-order convergence are derived in the literature. In this paper, we consider the fifth order method presented in [3], to study about their semi local convergence. Firstly, we have extend this method in Banach space, which is as follows

$$
\begin{aligned}
& y_{n}=x_{n}-\frac{1}{2} \Gamma_{n} T\left(x_{n}\right), \\
& z_{n}=x_{n}-\left[T^{\prime}\left(y_{n}\right)\right]^{-1} T\left(x_{n}\right), \\
& x_{n+1}=z_{n}-\left[2\left[T^{\prime}\left(Y_{n}\right)\right]^{-1}-\Gamma_{n}\right] T\left(z_{n}\right) .
\end{aligned}
$$

Here $\Gamma_{n}=\left[T^{\prime}\left(x_{n}\right)\right]^{-1}$. The technique of recurrence relations has been widely and successfully used in formulating the convergence of Newton's method and various higher order method for examples, we can see as $[[4],[5],[7],[8]-[15]]$ are few of them. Here, we use the recurrence relations to derived the semi local convergence for the considered method. We have constructed the system of recurrence relations and proved the convergence of method, along with an error estimate. In the end, some numerical results are introduced to demonstrate our approach.

Lemma 1. Assume that the nonlinear operator $T: \Omega \subseteq X \rightarrow Y$ is continuously second-order Fréchet differentiable, where $\Omega$ is open set and $X$ and $Y$ are Banach spaces. Then we can obtain

$$
\begin{aligned}
& T\left(x_{n+1}\right)=\frac{1}{2} T^{\prime \prime}\left(x_{n}\right)\left(w_{n}-x_{n}\right)\left[T^{\prime}\left(y_{n}\right)\right]^{-1}\left[T^{\prime \prime}\left(x_{n}\right)\left(w_{n}-x_{n}\right)\right. \\
& \left.+\int_{0}^{1}\left[T^{\prime \prime}\left(x_{n}+\frac{u}{2}\left(w_{n}-x_{n}\right)\right)-T^{\prime \prime}\left(x_{n}\right)\right] d u\left(w_{n}-x_{n}\right)\right] \Gamma_{n} T\left(z_{n}\right) \\
& +\int_{0}^{1}\left[T^{\prime \prime}\left(x_{n}+u\left(w_{n}-x_{n}\right)\right)-T^{\prime \prime}\left(x_{n}\right)\right]\left(w_{n}-x_{n}\right) d u\left(x_{n+1}-z_{n}\right) \\
& -\frac{1}{2} \int_{0}^{1}\left[T^{\prime \prime}\left(x_{n}+\frac{u}{2}\left(w_{n}-x_{n}\right)\right)-T^{\prime \prime}\left(x_{n}\right)\right]\left(w_{n}-x_{n}\right) d u\left(x_{n+1}-z_{n}\right) \\
& +\frac{1}{2} \int_{0}^{1}\left[T^{\prime \prime}\left(x_{n}+\frac{u}{2}\left(w_{n}-x_{n}\right)\right)-T^{\prime \prime}\left(x_{n}\right)\right] d u\left(w_{n}-x_{n}\right) \Gamma_{n} T\left(z_{n}\right) \\
& +\int_{0}^{1}\left[T^{\prime}\left(z_{n}+u\left(x_{n+1}-z_{n}\right)\right)-T^{\prime}\left(w_{n}\right)\right]\left(x_{n+1}-z_{n}\right) d u,
\end{aligned}
$$

where $w_{n}=x_{n}-\Gamma_{n} T\left(x_{n}\right)$. 
Proof. Since

$$
T^{\prime}\left(y_{n}\right)\left(x_{n+1}-z_{n}\right)=-\left[2 T^{\prime}\left(x_{n}\right)-T^{\prime}\left(y_{n}\right)\right] \Gamma_{n} T\left(z_{n}\right),
$$

where $\left[T^{\prime}\left(x_{n}\right)\right]^{-1}=\Gamma_{n}$. By Taylor expansion, we find

$$
\begin{aligned}
& T\left(x_{n+1}\right)=T\left(z_{n}\right)+T^{\prime}\left(w_{n}\right)\left(x_{n+1}-z_{n}\right) \\
& +\int_{0}^{1}\left[T^{\prime}\left(z_{n}+u\left(x_{n+1}-z_{n}\right)\right)-T^{\prime}\left(w_{n}\right)\right]\left(x_{n+1}-z_{n}\right) d u \\
& =T\left(z_{n}\right)+\left[T^{\prime}\left(w_{n}\right)-T^{\prime}\left(y_{n}\right)\right]\left(x_{n+1}-z_{n}\right) \\
& -\left[2 T^{\prime}\left(x_{n}\right)-T^{\prime}\left(y_{n}\right)\right] \Gamma_{n} T\left(z_{n}\right) \\
& +\int_{0}^{1}\left[T^{\prime}\left(z_{n}+u\left(x_{n+1}-z_{n}\right)\right)-T^{\prime}\left(w_{n}\right)\right]\left(x_{n+1}-z_{n}\right) d u
\end{aligned}
$$

Similarly, we have

$$
\begin{aligned}
T^{\prime}\left(w_{n}\right) & =T^{\prime}\left(x_{n}\right)+T^{\prime \prime}\left(x_{n}\right)\left(w_{n}-x_{n}\right) \\
& +\int_{0}^{1}\left[T^{\prime \prime}\left(x_{n}+u\left(w_{n}-x_{n}\right)\right)-T^{\prime \prime}\left(x_{n}\right)\right]\left(w_{n}-x_{n}\right) d u
\end{aligned}
$$

and

$$
\begin{aligned}
T^{\prime}\left(y_{n}\right) & =T^{\prime}\left(x_{n}\right)+\frac{1}{2} T^{\prime \prime}\left(x_{n}\right)\left(w_{n}-x_{n}\right) \\
& +\frac{1}{2} \int_{0}^{1}\left[T^{\prime \prime}\left(x_{n}+\frac{u}{2}\left(w_{n}-x_{n}\right)\right)-T^{\prime \prime}\left(x_{n}\right)\right]\left(w_{n}-x_{n}\right) d u
\end{aligned}
$$

Here it follows that

$$
\begin{aligned}
& T^{\prime}\left(w_{n}\right)-T^{\prime}\left(y_{n}\right)=\frac{1}{2} T^{\prime \prime}\left(x_{n}\right)\left(w_{n}-x_{n}\right) \\
& +\int_{0}^{1}\left[T^{\prime \prime}\left(x_{n}+u\left(w_{n}-x_{n}\right)\right)-T^{\prime \prime}\left(x_{n}\right)\right]\left(w_{n}-x_{n}\right) d u \\
& -\frac{1}{2} \int_{0}^{1}\left[T^{\prime \prime}\left(x_{n}+\frac{u}{2}\left(w_{n}-x_{n}\right)\right)-T^{\prime \prime}\left(x_{n}\right)\right]\left(w_{n}-x_{n}\right) d u .
\end{aligned}
$$

Now, we can calculate

$$
\begin{aligned}
& {\left[2 T^{\prime}\left(x_{n}\right)-T^{\prime}\left(y_{n}\right)\right]=T^{\prime}\left(x_{n}\right)-\frac{1}{2} T^{\prime \prime}\left(x_{n}\right)\left(w_{n}-x_{n}\right)} \\
& -\frac{1}{2} \int_{0}^{1}\left[T^{\prime \prime}\left(x_{n}+\frac{u}{2}\left(w_{n}-x_{n}\right)\right)-T^{\prime \prime}\left(x_{n}\right)\right]\left(w_{n}-x_{n}\right) d u .
\end{aligned}
$$

By considering (3), (4) and (5), we obtain (2). 
Now, we study some properties of the following scalar functions which will be frequently used in the later results:

$$
\begin{gathered}
p(u)=\frac{u(4-u)(6-u)}{(2-u)^{3}}+\frac{2}{(2-u)}, \\
\delta(u)=\frac{1}{1-u p(u)}, \\
\varphi(u, v)+\left[\frac{u}{(2-u)}(u+v)+\frac{v}{8}\right]\left[\frac{1}{2} \frac{u^{3}}{(2-u)^{2}}+\frac{u^{2}}{2(2-u)}+\frac{7}{24} v\right] \\
+\frac{5 v}{8} \frac{(4-u)}{(2-u)}\left[\frac{1}{2} \frac{u^{3}}{(2-u)^{2}}+\frac{u^{2}}{(2-u)}+\frac{7 v}{24}\right] \\
+\frac{u^{2}(4-u)}{(2-u)^{2}}\left[\frac{1}{2} \frac{u^{3}}{(2-u)^{2}}+\frac{u^{2}}{(2-u)}+\frac{7}{24} v\right] \\
+\frac{u(4-u)^{2}}{2(2-u)^{2}}\left[\frac{1}{2} \frac{u^{3}}{(2-u)^{2}}+\frac{u^{2}}{(2-u)}+\frac{7}{24} v\right]^{2} .
\end{gathered}
$$

Let $q(u)=u p(u)-1$, and $q(0)=-1$ and $q(2)=+\infty$ so $q(u)$ has at least a zero in $(0,2)$. Let $\phi$ is a smallest positive zero of the scalar function $q(u)$. Some properties of the functions defined above are given in the following lemmas.

Lemma 2. Suppose the scalar functions $p, \delta$ and $\varphi$ be given in (6), (7) and (8), respectively then

(a) $p(u)$ and $\delta(u)$ are increasing and $p(u)>1, \delta(u)>1$ for $u \in(0, \phi)$,

(b) $\varphi(u, v)$ is increasing for $u \in(0, \phi), v>0$.

Let the condition $(C 1)-(C 4)$ hold. Now, we mention $\rho_{0}=\rho, \tau_{0}=\tau$, $\alpha_{0}=K \tau_{0} \rho_{0}, \beta_{0}=L \tau_{0} \rho_{0}^{2}$, and $r_{0}=\delta\left(\alpha_{0}\right) \varphi\left(\alpha_{0}, \beta_{0}\right)$. Moreover, we can define the following sequences for $n \geq 0$

$$
\begin{gathered}
\rho_{n+1}=r_{n} \rho_{n}, \\
\tau_{n+1}=\delta\left(\alpha_{n}\right) \tau_{n}, \\
\alpha_{n+1}=K \tau_{n+1} \rho_{n+1}, \\
\beta_{n+1}=L \tau_{n+1} \rho_{n+1}^{2},
\end{gathered}
$$




$$
r_{n+1}=\delta\left(\alpha_{n}\right) \varphi\left(\alpha_{n+1}, \beta_{n+1}\right)
$$

From the definition of $\alpha_{n+1}, \beta_{n+1}$ and (9)-(12), we also have

$$
\begin{aligned}
\alpha_{n+1} & =\delta\left(\alpha_{n}\right) r_{n} \alpha_{n} \\
\beta_{n+1} & =\delta\left(\alpha_{n}\right) r_{n}^{2} \beta_{n} .
\end{aligned}
$$

Lemma 3. Assume the real functions $p, \delta$ and $\varphi$ are defined as in (6), (7) and (8), respectively and

$$
0<\alpha_{0}<\phi, \delta\left(\alpha_{0}\right) r_{0}<1
$$

then we have

(a) $\delta\left(\alpha_{n}\right)>1$ and $\delta_{n} \varphi\left(\alpha_{n}, \beta_{n}\right)<1$ for $n \geq 0$,

(b) the sequences $\left\{\rho_{n}\right\},\left\{\alpha_{n}\right\},\left\{\beta_{n}\right\}$ and $\left\{r_{n}\right\}$ are decreasing,

(c) $p\left(\alpha_{n}\right) \alpha_{n}<1$ and $\delta\left(\alpha_{n}\right) r_{n}<1$ for $n \geq 0$.

Proof. From the previous Lemma 2 and the equation (14), $\delta\left(\alpha_{0}\right)>1$ and $\delta_{n} \varphi\left(\alpha_{n}, \beta_{n}\right)<1$ hold. It follows from the equations (9), (11) and (12) that $\rho_{1}<\rho_{0}, \alpha_{1}<\alpha_{0}, \beta_{1}<\beta_{0}$, for $n \geq 0$. Furthermore, by Lemma 2 , we can get $\delta\left(\alpha_{1}\right)>\delta\left(\alpha_{0}\right)$ and $\varphi\left(\alpha_{1}, \beta_{1}\right)<\varphi\left(\alpha_{0}, \beta_{0}\right)$. This follow $r_{1}<r_{0}$ and (b) holds. Similarly, we obtain these results $p\left(\alpha_{1}\right) \alpha_{1}<p\left(\alpha_{0}\right) \alpha_{0}<1$ and $\delta\left(\alpha_{1}\right) r_{1}<$ $\delta\left(\alpha_{0}\right) r_{0}<1$ and (c) holds for all $n \geq 0$. Using mathematical induction, we can determine that (a), (b) and (c).

Lemma 4. Assume the real functions $p, \delta$ and $\varphi$ be given in (6)-(8) and let $\xi \in(0,1)$, then $p(\xi u)<p(u), \delta(\xi u)<\delta(u)$ and $\varphi\left(\xi u, \xi^{2} v\right)<\xi^{4} \varphi(u, v)$ for $u \in(0, \phi)$.

Proof. The proof is straight forward.

Lemma 5. Following the assumptions of Lemma 3. Let $\zeta=\delta^{2}\left(\alpha_{0}\right) \varphi\left(\alpha_{0}, \beta_{0}\right)$, then

$$
r_{n} \leq \lambda \zeta^{5^{n}}, n \geq 0
$$

where $\lambda=1 / \delta\left(\alpha_{0}\right)$. Also for $n \geq 0$, we have

$$
\prod_{i=0}^{n} \delta\left(\alpha_{i}\right) \varphi\left(\alpha_{i}, \beta_{i}\right) \leq \lambda^{n+1} \zeta \frac{5^{n+1}-1}{4}
$$


Proof. We know $\alpha_{1}=\delta\left(\alpha_{0}\right) r_{0} \alpha_{0}=\delta^{2}\left(\alpha_{0}\right) \varphi\left(\alpha_{0}, \beta_{0}\right) \alpha_{0}=\zeta \alpha_{0}$, Similarly, $\beta_{1}=\delta\left(\alpha_{0}\right) r_{0}^{2} \beta_{0}<\zeta^{2} \beta_{0}$. By induction theorem, $\delta\left(\alpha_{1}\right) \varphi\left(\alpha_{1}, \beta_{1}\right)<\delta\left(\zeta \alpha_{0}\right) \varphi\left(\zeta \alpha_{0}, \zeta^{2} \beta_{0}\right)<\zeta^{5^{1}-1} \delta\left(\alpha_{0}\right) \varphi\left(\alpha_{0}, \beta_{0}\right)=\lambda \zeta^{5^{1}}$. Let $\delta\left(\alpha_{n}\right) \varphi\left(\alpha_{n}, \beta_{n}\right) \leq \lambda \zeta^{5^{n}}, n \geq 1$. By considering Lemma 3 , we have

$$
\begin{aligned}
& \delta\left(\alpha_{n+1}\right) \varphi\left(\alpha_{n+1}, \beta_{n+1}\right) \leq \delta\left(\alpha_{n}\right) \varphi\left(\delta\left(\alpha_{n}\right) r_{n} \alpha_{n}, \delta\left(\alpha_{n}\right) r_{n}^{2} \beta_{n}\right) \\
\leq & \delta\left(\alpha_{n}\right) \varphi\left(\delta^{2}\left(\alpha_{n}\right) \varphi\left(\alpha_{n}, \beta_{n}\right) \alpha_{n}, \delta^{3}\left(\alpha_{n}\right) \varphi^{2}\left(\alpha_{n}, \beta_{n}\right) \beta_{n}\right) \\
\leq & \delta\left(\alpha_{n}\right) \varphi\left(\delta^{2}\left(\alpha_{n}\right) \varphi\left(\alpha_{n}, \beta_{n}\right) \alpha_{n}, \delta^{4}\left(\alpha_{n}\right) \varphi^{2}\left(\alpha_{n}, \beta_{n}\right) \beta_{n}\right) \\
\leq & \delta\left(\alpha_{n}\right)\left[\delta^{8}\left(\alpha_{n}\right) \varphi^{5}\left(\alpha_{n}, \beta_{n}\right)\right] \leq \lambda \zeta^{5^{n+1}} .
\end{aligned}
$$

Consequently, it holds that $r_{m} \leq \lambda \zeta^{5^{m}}, m \geq 0$. From (15), we have

$$
\prod_{i=0}^{m} \delta\left(\alpha_{i}\right) \varphi\left(\alpha_{i}, \beta_{i}\right) \leq \prod_{i=0}^{m} \lambda \zeta^{5^{i}}=\lambda^{m+1} \zeta^{\frac{\left(5^{n+1}-1\right)}{4}}, m \geq 0 .
$$

Lemma 6. Consider the assumptions of Lemma 3 and Lemma 5. The decreasing sequence $\left\{\rho_{n}\right\}$ satisfies

$$
\rho_{n} \leq \lambda^{n} \zeta^{\frac{5^{n}-1}{4}}, n \geq 0
$$

So the decreasing sequence $\left\{\rho_{n}\right\}$ converges to zero. Furthermore, for any $n \geq 0$, $m \geq 1$, it holds

$$
\sum_{i=n}^{n+m} \rho_{i} \leq \rho \lambda^{n} \zeta^{\frac{5^{n}-1}{4}}\left(\frac{1-\lambda^{m+1} \zeta^{\frac{5^{n}\left(5^{m}+3\right)}{4}}}{1-\lambda \zeta^{5^{n}}}\right) .
$$

Proof. Using the equations (9) and (15), the sequence $\left\{\rho_{n}\right\}$ can be converted into a form

$$
\begin{aligned}
\rho_{n} & =\delta\left(\alpha_{n-1}\right) \varphi\left(\alpha_{n-1}, \beta_{n-1}\right) \rho_{n-1} \\
& =\delta\left(\alpha_{n-1}\right) \varphi\left(\alpha_{n-1}, \beta_{n-1}\right) \rho_{n-2} \delta\left(\alpha_{n-2}\right) \varphi\left(\alpha_{n-2}, \beta_{n-2}\right)=\ldots \\
& =\rho\left(\prod_{i=0}^{n-1} \delta\left(\alpha_{i}\right) \varphi\left(\alpha_{i}, \beta_{i}\right)\right) \leq \rho \lambda^{n} \zeta^{\frac{\left(5^{n}-1\right)}{4}} .
\end{aligned}
$$

Because $\lambda<1$ and $\zeta<1$, it follows that $\rho_{n} \rightarrow 0$ as $n \rightarrow \infty$; which show that the sequence $\left\{\rho_{n}\right\}$ converges to zero. When $n \geq 0, m \geq 1$,

$$
\theta \quad=\Sigma_{i=n}^{n+m} \lambda^{i} \zeta^{5^{i} / 4}
$$




$$
\begin{aligned}
& =\lambda^{n} \zeta^{5^{n} / 4}+\sum_{i=n+1}^{n+m} \lambda^{i} \zeta^{5^{i} / 4} \\
& =\lambda^{n} \zeta^{5^{n} / 4}+\lambda \zeta^{5^{n}}\left(\sum_{i=n+1}^{n+m-1} \lambda^{i} \zeta^{5^{i} / 4}\right) \\
& \leq \lambda^{n} \zeta^{5^{n} / 4}+\lambda^{n} \zeta^{5^{n}} \theta-\lambda^{n+m+1} \zeta^{5^{n}\left(4+5^{m}\right) / 4} \\
& \leq \lambda^{n} \zeta^{\frac{5^{n}}{4}}\left(\frac{1-\lambda^{m+1} \zeta^{\frac{5^{n}\left(5^{m}+3\right)}{4}}}{1-\lambda \zeta^{5^{n}}}\right)
\end{aligned}
$$

Furthermore

$$
\begin{aligned}
\sum_{i=n}^{n+m} \rho_{i} & \leq \rho \sum_{i=n}^{n+m} \lambda^{i} \zeta^{\frac{5^{n}-1}{4}}, \text { for } n \geq 0 \\
& =\rho \zeta^{-1 / 4} \theta \\
& \leq \rho \lambda^{n} \zeta^{\left(5^{n}-1\right) / 4}\left(\frac{1-\lambda^{m+1} \zeta^{\frac{5^{n}\left(5^{m}+4\right)}{4}}}{1-\lambda \zeta^{5^{n}}}\right)
\end{aligned}
$$

Therefore, $\Sigma_{n=0}^{\infty} \rho_{n}$ exists. And thus the proof is completed.

\section{Recurrence Relations for the Method}

We mention $B(x, \kappa)=\{y \in X:\|y-x\|<\kappa\}$ and $\overline{B(x, \kappa)}=\{y \in X:\|y-x\| \leq$ $\kappa\}$ in this paper. Under the previous assumtion, we have derived the following recurrence relations for the method.

For $n=0$, the existence of $\Gamma_{0}$ implies the existence of $w_{0}, y_{0}$ and $\kappa_{0}$. Then, we obtain

$$
\left\|w_{0}-x_{0}\right\|=\left\|\zeta_{0} T\left(x_{0}\right)\right\| \leq \rho_{0} .
$$

and

$$
\left\|y_{0}-x_{0}\right\| \leq \frac{\rho_{0}}{2}
$$

This means that $w_{0}, y_{0} \in B\left(x_{0}, R \rho\right)$, where $R=\frac{p\left(\alpha_{0}\right)}{1-r_{0}}$. let $H\left(x_{0}\right)=\Gamma_{0}\left[T^{\prime}\left(x_{0}\right)-\right.$ $\left.T^{\prime}\left(y_{0}\right)\right]$, then

$$
\left\|H\left(x_{0}\right)\right\| \leq K \tau\left\|y_{0}-x_{0}\right\| \leq \frac{\alpha_{0}}{2} .
$$

Using Banach lemma it follows that $\left[I+H\left(x_{0}\right)\right]^{-1}$ exists and satisfies

$$
\left\|\left[I+H\left(x_{0}\right)\right]^{-1}\right\| \leq \frac{2}{2-\alpha_{0}}, \text { if } \alpha_{0}<2 .
$$

Consequently, we obtain

$$
\left\|z_{0}-x_{0}\right\| \leq \frac{2}{\left(2-\alpha_{0}\right)} \rho_{0} .
$$


Similarly

$$
\left\|z_{0}-w_{0}\right\| \leq \frac{\alpha_{0}}{2-\alpha_{0}} \rho_{0}
$$

From the Banach lemma, we have that $\left[T^{\prime}\left(y_{0}\right)\right]^{-1}$ exists and

$$
\left\|\left[T^{\prime}\left(y_{0}\right)\right]^{-1}\right\| \leq \frac{\tau_{0}}{1-\frac{1}{2} \alpha_{0}} .
$$

Since

$$
\begin{aligned}
\left\|T\left(z_{0}\right)\right\| & \leq\left\|I+\left[T^{\prime}\left(y_{0}\right)\right]^{-1}\left[T^{\prime}\left(x_{0}\right)-T^{\prime}\left(y_{0}\right)\right]\right\| \\
& \left\|T^{\prime}\left(x_{0}\right)-T^{\prime}\left(y_{0}\right)\right\|\left\|\Gamma_{0} T\left(x_{0}\right)\right\| \\
& +\| \int_{0}^{1}\left[T^{\prime}\left(x_{0}+u\left(z_{0}-x_{0}\right)-T^{\prime}\left(x_{0}\right)\right] d u\left(z_{0}-x_{0}\right) \|\right. \\
& \leq \frac{K \rho_{0}^{2}}{2-\alpha_{0}}+\frac{2 K \rho_{0}^{2}}{\left(2-\alpha_{0}\right)^{2}} .
\end{aligned}
$$

Hence, we have

$$
\left\|x_{1}-z_{0}\right\| \leq \frac{\left(6-\alpha_{0}\right)}{\left(2-\alpha_{0}\right)} \tau_{0}\left\|T\left(z_{0}\right)\right\|
$$

and

$$
\left\|x_{1}-x_{0}\right\| \leq\left\|x_{1}-z_{0}\right\|+\left\|z_{0}-x_{0}\right\| \leq p\left(\alpha_{0}\right) \rho_{0} .
$$

Under the assumption $r_{0}<1 / \delta\left(\alpha_{0}\right)<1$, it follows that $x_{1} \in B\left(x_{0}, R \rho\right)$. By $\alpha_{0} \leq \phi$ and $p\left(\alpha_{0}\right) \leq p(\phi)$, we have

$$
\begin{aligned}
\left\|I-\Gamma_{0} F^{\prime}\left(x_{1}\right)\right\| & \leq\left\|\zeta_{0}\right\|\left\|F^{\prime}\left(x_{0}\right)-F^{\prime}\left(x_{1}\right)\right\| \\
& \leq K \tau_{0}\left\|x_{1}-x_{0}\right\| \\
& \leq \alpha_{0} p\left(\alpha_{0}\right)<1 .
\end{aligned}
$$

Furthermore

$$
\left\|\Gamma_{1}\right\| \leq \frac{\tau_{0}}{\left(1-\alpha_{0} p\left(\alpha_{0}\right)\right)}=\delta\left(\alpha_{0}\right) \tau_{0}=\tau_{1}<1
$$

We now consider $T\left(z_{0}\right)$. From the results in [13]

$$
T\left(z_{n}\right)=\int_{0}^{1} T^{\prime \prime}\left(w_{n}+u\left(z_{n}-w_{n}\right)\right)(1-u) d u\left(z_{n}-w_{n}\right)^{2}
$$




$$
\begin{aligned}
& -\int_{0}^{1} T^{\prime \prime}\left(y_{n}+u\left(w_{n}-y_{n}\right)\right)\left(w_{n}-y_{n}\right) d u \\
& {\left[T^{\prime}\left(y_{n}\right)\right]^{-1}\left[T^{\prime}\left(y_{n}\right)-T^{\prime}\left(x_{n}\right)\right]\left(w_{n}-x_{n}\right)} \\
& +\int_{0}^{1}\left[T^{\prime \prime}\left(x_{n}+u\left(w_{n}-x_{n}\right)\right)-T^{\prime \prime}\left(x_{n}\right)\right](1-u) d u\left(w_{n}-x_{n}\right)^{2} \\
& +\frac{1}{2} \int_{0}^{1}\left[T^{\prime \prime}\left(x_{n}\right)-T^{\prime \prime}\left(x_{n}+\frac{1}{2} u\left(w_{n}-x_{n}\right)\right] d u\left(w_{n}-x_{n}\right)^{2}\right.
\end{aligned}
$$

and hence, we can obtain

$$
\begin{aligned}
\left\|T\left(z_{0}\right)\right\| & \leq \frac{K}{2}\left\|z_{0}-w_{0}\right\|^{2} \\
& +K^{2}\left\|T^{\prime}\left(y_{0}\right)^{-1}\right\|\left\|w_{0}-y_{0}\right\|\left\|y_{0}-x_{0}\right\|\left\|w_{0}-x_{0}\right\| \\
& +\frac{7}{24} L\left\|w_{0}-x_{0}\right\|^{3} \\
& \leq \frac{K}{2} \frac{\alpha_{0}^{2} \rho_{0}^{2}}{\left(2-\alpha_{0}\right)^{2}}+K^{2} \frac{2 \beta_{0}}{\left(2-\alpha_{0}\right)} \frac{\rho_{0}^{3}}{4}+\frac{7}{24} L \rho_{0}^{3} .
\end{aligned}
$$

Therefore, we have

$$
\begin{aligned}
\left\|x_{1}-z_{0}\right\| & \leq \frac{6-\alpha_{0}}{2-\alpha_{0}} \tau_{0}\left\|T\left(z_{0}\right)\right\| \\
& \leq \frac{6-\alpha_{0}}{2-\alpha_{0}}\left[\frac{1}{2} \frac{\alpha_{0}^{3}}{\left(2-\alpha_{0}\right)^{2}}+\frac{\alpha_{0}^{2}}{2\left(2-\alpha_{0}\right)}+\frac{7}{24} \beta_{0}\right] \rho_{0} .
\end{aligned}
$$

By Lemma 1, we have

$$
\begin{aligned}
\left\|T\left(x_{1}\right)\right\| \leq & {\left[\frac{\alpha_{0}}{2-\alpha_{0}}\left(\alpha_{0}+\beta_{0}\right)+\frac{\beta_{0}}{8}\right]\left\|T\left(z_{0}\right)\right\|+\frac{5}{8} L \rho_{0}^{2}\left\|x_{1}-z_{0}\right\| } \\
& +K\left\|z_{0}-w_{0}\right\|\left\|x_{1}-z_{0}\right\|+\frac{K}{2}\left\|x_{1}-z_{0}\right\|^{2} .
\end{aligned}
$$

Considering the equations (35) and (39), it follows that

$$
\begin{aligned}
\left\|w_{1}-x_{1}\right\| & \leq\left\|\Gamma_{1} T\left(x_{1}\right)\right\| \\
& \leq \delta\left(\alpha_{0}\right) \varphi\left(\alpha_{0}, \beta_{0}\right) \rho_{0} \\
& =r_{0} \rho_{0}=\rho_{1} .
\end{aligned}
$$

We know that $g\left(\alpha_{0}\right)>1$, then

$$
\left\|w_{1}-x_{0}\right\| \leq\left\|w_{1}-x_{1}\right\|+\left\|x_{1}-x_{0}\right\|
$$




$$
<p\left(\alpha_{0}\right)\left(1+r_{0}\right) \rho<R \rho
$$

which gives $w_{1} \in B\left(x_{0}, R \rho\right)$. We find

$$
\begin{aligned}
& K\left\|\Gamma_{1}\right\|\left\|\Gamma_{1} T\left(x_{1}\right)\right\| \leq \delta\left(\alpha_{0}\right) r_{0} \alpha_{0}=\alpha_{1}, \\
& L\left\|\Gamma_{1}\right\|\left\|\Gamma_{1} T\left(x_{1}\right)\right\|^{2} \leq \delta\left(\alpha_{0}\right) r_{0}^{2} \beta_{0}=\beta_{1} .
\end{aligned}
$$

Repeating this procedure, we can the system of recurrence relations given in the next lemma:

Lemma 7. Consider the assumptions of Lemma 3 and the conditions $(C 1)-(C 4)$ hold. Then the following relations are true for all $n \geq 0$ :

(I) There exists $\Gamma_{n}=\left[T^{\prime}\left(x_{n}\right)\right]^{-1}$ and $\left\|\Gamma_{n}\right\| \leq \tau_{n}$,

(II) $\left\|\Gamma_{n} T\left(x_{n}\right)\right\| \leq \rho_{n}$,

(III) $K\left\|\Gamma_{n}\left|\left\||| \Gamma_{n} T\left(x_{n}\right)\right\| \leq \alpha_{n}\right.\right.$,

(IV) $L\left\|\Gamma_{n}\right\|\left\|\Gamma_{n} T\left(x_{n}\right)\right\|^{2} \leq \beta_{n}$,

(V) $\left\|x_{n+1}-x_{n}\right\| \leq p\left(\alpha_{n}\right) \rho_{n}$,

(VI) $\left\|x_{n+1}-x_{0}\right\| \leq R \rho$, where $R=\frac{p\left(\alpha_{0}\right)}{1-\delta\left(\alpha_{0}\right) \varphi\left(\alpha_{0}, \beta_{0}\right)}$.

Proof. The proof of $(I)-(V)$ follows by using previous results and by mathematical hypothesis. We only consider $(V I)$. By $(V)$ and Lemma 6, we have

$$
\begin{aligned}
\left\|x_{n+1}-x_{0}\right\| & \leq \Sigma_{i=0}^{n}\left\|x_{i+1}-x_{i}\right\| \\
& \leq \Sigma_{i=0}^{n} p\left(\alpha_{i}\right) \rho_{i} \\
& \leq p\left(\alpha_{0}\right) \frac{1-\lambda^{n+1} \zeta^{\left(5^{n}+3\right) / 4}}{1-\delta\left(\alpha_{0}\right) \varphi\left(\alpha_{0}, \beta_{0}\right)}<R \rho .
\end{aligned}
$$

Hence, this result proved.

\section{Semilocal Convergence}

In this section, we have studied the theorem for the semilocal convergence of (1.2), This theorem defines existence and uniqueness of the solution and find a periori error bounds. 
Theorem 8. Assume $T: \Omega \subseteq X \rightarrow Y$ be a nonlinear second order Fréchet differential operator in an open convex subset $\Omega$ of a Banach space $X$ to $Y$. Suppose $x_{0} \in \Omega$ and all relations $(C 1)-(C 4)$ hold. Let $\alpha_{0}=K \tau \rho, \beta_{0}=L \tau \rho^{2}$, and $r_{0}=\delta\left(\alpha_{0}\right) \varphi\left(\alpha_{0}, \beta_{0}\right)$ satisfy $\alpha_{0}<\phi$ and $\delta\left(\alpha_{0}\right) r_{0}<1$, where $p, \delta$ and $\varphi$ are defined by (6)-(8). Let $B\left(x_{0}, R \rho\right) \subset \Omega$, where $R=p\left(\alpha_{0}\right) /\left(1-\delta\left(\alpha_{0}\right) \varphi\left(\alpha_{0}, \beta_{0}\right)\right.$, initializing from $x_{0}$, the sequence $x_{n}$ generated by the method converges to a solution $x^{*}$ of $T(x)=0$ with $x_{n}, x^{*}$ belonging to $B\left(x_{0}, R \rho\right)$ and $x^{*}$ belong the unique solution of $T(x)=0$ in $B\left(x_{0},(2 / K \tau-R \rho) \bigcap \Omega\right.$. Furthermore, a priori error estimate is defined by

$$
\left\|x_{n}-x^{*}\right\| \leq \frac{\left(p\left(\alpha_{0}\right) \rho \lambda^{n} \zeta^{\left(5^{n}-1\right) / 4}\right)}{1-\lambda \zeta^{5^{n}}}
$$

where $\zeta=\delta\left(\alpha_{0}\right) r_{0}$ and $\zeta=1 / \delta\left(\alpha_{0}\right)$.

Proof. In Lemma $7, x_{n}$ is well defined sequence in $B\left(x_{0}, R \rho\right)$. Here, we show that $x_{n}$ is a Cauchy sequence. Since

$$
\begin{aligned}
\left\|x_{n+m}-x_{n}\right\| & \leq \sum_{i=n}^{n+m-1}\left\|x_{i+1}-x_{i}\right\| \\
& \leq \sum_{i=n}^{n+m-1} p\left(\alpha_{i}\right) \rho_{i} \\
& \leq p\left(\alpha_{0}\right) \rho \lambda^{n} \zeta^{\frac{5^{n}-1}{4}}\left(\frac{1-\lambda^{m+1} \zeta^{\frac{5^{n}\left(5^{m}+3\right)}{4}}}{1-\lambda \zeta^{5^{n}}}\right) .
\end{aligned}
$$

Hence $x_{n}$ is a Cauchy sequence. So there exists a $x^{*}$ such that $\lim _{n \rightarrow \infty} x_{n}=x^{*}$. Assuming $n=0, m \rightarrow \infty$ in, we have

$$
\left\|x_{n+m}-x_{n}\right\| \leq R \rho
$$

This proved $x^{*} \in B\left(x_{0}, R \rho\right)$. Again, we show that $x^{*}$ is solution of $T(x)=0$. Since

$$
\left\|\Gamma _ { 0 } \left|\left\||| T\left(x_{0}\right)\right\| \leq\left\|\Gamma_{n}\right\|\left\|T\left(x_{n}\right)\right\| \leq \rho_{n}\right.\right.
$$

By letting $n \rightarrow \infty$ in (47), we find $\left\|T\left(x_{n}\right)\right\| \rightarrow 0$ since $p\left(\alpha_{n}\right)<p\left(\alpha_{0}\right)$ and $\rho_{n} \rightarrow 0$. Hence by the definition of continuity $T$ in $\Omega$, we find $T\left(x^{*}\right)=0$. Now, we show the uniqueness of $x^{*}$ in $B\left(x_{0}, \frac{2}{K \tau}-R \rho\right) \bigcap \Omega$. First of all we can obtain $x^{*} \in B\left(x_{0}, \frac{2}{K \tau}-R \rho\right) \bigcap \Omega$, it follows

$$
\frac{2}{K \tau}-R \rho=\left(\frac{2}{\alpha_{0}}-R\right) \rho>R \rho,
$$


and then $B\left(x_{0}, R \rho\right) \subset B\left(x_{0}, 2 / K \tau-R \rho\right) \bigcap \Omega$. Let $x^{* *}$ be second zero of $T(x)$ in $B\left(x_{0}, 2 / K \tau-R \rho\right) \bigcap \Omega$. By Taylor theorem, we have

$$
0=T\left(x^{* *}\right)-T\left(x^{*}\right)=\int_{0}^{1} T^{\prime}\left((1-u) x^{*}+u x^{* *}\right) d u\left(x^{* *}-x^{*}\right) .
$$

Since

$$
\begin{aligned}
& \left\|\Gamma_{0}\right\|\left\|\int_{0}^{1}\left[T^{\prime}\left((1-u) x^{*}+u x^{* *}\right)-T^{\prime}\left(x_{0}\right)\right] d u\right\| \\
& \leq K \tau \int_{0}^{1}\left[(1-u)\left\|x^{*}-x_{0}\right\|+u\left\|x^{* *}-x_{0}\right\|\right] d u \\
& \leq \frac{K \tau}{2}\left[R \rho+\frac{2}{K \tau}-R \rho\right]=1 .
\end{aligned}
$$

Using Banach lemma that $\int_{0}^{1} T^{\prime}\left((1-u) x^{*}+u x^{* *}\right) d u$ is invertible and hence $x^{* *}=x^{*}$. Now, by letting $m \rightarrow \infty$ in (45), we have

$$
\left\|x_{n}-x^{*}\right\| \leq \frac{p\left(\alpha_{0}\right) \rho}{\zeta^{1 / 4}\left(1-r_{0}\right)}\left(\zeta^{1 / 4}\right)^{5^{n}}
$$

These result shows that the method given by is of $R$ - order of convergence at least five. Thus proof is completed.

\section{Numerical Result}

Now, consider the nonlinear integral equation $T(x)=0$, where

$$
T(x)(\phi)=x(\phi)-1-\frac{1}{2} \int_{0}^{1} \phi \cos (x(\phi)) d u,
$$

and $\phi \in[0,1]$ for $x \in \Omega=B(0,2) \subset X$. Let $X=C[0,1]$ is the space of continuous functions which is defined for $[0,1]$. We can easily find the derivative of $T$ as

$$
T^{\prime}(x) y(\phi)=y(\phi)-\frac{1}{2} \int_{0}^{1} \phi \sin (x(u)) y(u) d u, y \in \Omega,
$$

and

$$
T^{\prime \prime}(x) y z(\phi)=-\frac{1}{2} \int_{0}^{1} \phi \cos (x(u)) y(u) z(u) d u, y, z \in \Omega .
$$


Now, we can obtain

$$
\left\|T^{\prime \prime}(x)\right\| \leq \frac{1}{2}=K
$$

By Lipschitz condition with, $L=\frac{1}{2}$

$$
\left\|T^{\prime \prime}(x)-T^{\prime \prime}(y)\right\| \leq \frac{1}{2}\|x-y\|, x, y \in \Omega .
$$

The value of a scalar function $x_{0}=\frac{4}{3}$, is consider as the initial approximate solution. It follow that

$$
\left\|T^{\prime \prime}\left(x_{0}\right)\right\| \leq \frac{1}{2} \cos \frac{4}{3}
$$

Here, we have

$$
\left\|I-T^{\prime}\left(x_{0}\right)\right\| \leq \frac{1}{2} \sin \frac{4}{3},
$$

From the Banach lemma, we conclude that $\Gamma_{0}$ exists and satisfies

Table 1: Results of recurrence relations

\begin{tabular}{llllll}
\hline $\mathrm{n}$ & $\rho_{\mathrm{n}}$ & $\tau_{\mathrm{n}}$ & $\alpha_{\mathrm{n}}$ & $\beta_{\mathrm{n}}$ & $r_{\mathrm{n}}$ \\
\hline 0 & 0.228 & 1.945 & 0.222 & 0.050 & 0.009 \\
1 & 0.002 & 3.492 & 0.003 & $8.387 * 10^{-6}$ & $1.912 * 10^{-10}$ \\
2 & $4.191 * 10^{-13}$ & 3.506 & $7.3474 * 10^{-13}$ & $3.079 * 10^{-25}$ & $2.563 * 10^{-49}$ \\
3 & $1.074 * 10^{-61}$ & 3.506 & $1.883 * 10^{-61}$ & $2.024 * 10^{-122}$ & $1.107 * 10^{-243}$ \\
4 & $1.190 * 10^{-304}$ & 3.506 & $2.087 * 10^{-304}$ & $20484 * 10^{-608}$ & $1.669 * 10^{-1215}$ \\
5 & $1.987 * 10^{-1519}$ & 3.506 & $3.483 * 10^{-1519}$ & $6.921 * 10^{-3038}$ & $1.295 * 10^{-6074}$ \\
\hline
\end{tabular}

$$
\left\|\Gamma_{0}\right\| \leq \frac{2}{2-\sin \frac{4}{3}} \equiv \tau,
$$

Furthermore, we have

$$
\| \Gamma_{0} T\left(x_{0}\right) \leq \frac{\cos \frac{4}{3}}{2-\sin \frac{4}{3}} \equiv \rho,
$$

It also follows that

$$
\begin{aligned}
& \alpha_{0}=K \tau \rho=\frac{\cos \frac{4}{3}}{\left(2-\sin \frac{4}{3}\right)^{2}}, \\
& \beta_{0}=L \tau \rho^{2}=\frac{\cos ^{2} \frac{4}{3}}{\left(2-\sin \frac{4}{3}\right)^{3}},
\end{aligned}
$$


Now, we can obtain

$$
q\left(\alpha_{0}\right)=\alpha_{0} p\left(\alpha_{0}\right)-1=-0.557033 \leq 0,
$$

and

$$
r_{0} \delta\left(\alpha_{0}, \beta_{0}\right)=0.00961525 \leq 1
$$

Thus assumptions of the Theorem 8 are satisfied and recurrence relations for the presented method are shown in Table 1. Other than the solution $x^{*}$ belongs to $B\left(x_{0}, R \rho\right)=B\left(\frac{4}{3}, 0.00224254\right) \subset \Omega$ and it is unique in $B\left(\frac{4}{3}, 1.14309\right) \cap \Omega$.

\section{References}

[1] L. V. Kantorovich, G. P. Akilov, F. Ahmad, Functional analysis, Pergamon Press, Oxford (1982).

[2] L. B. Rall, Computational solution of nonlinear operator equations, In: Robert E. Krieger (ed.), New York (1979).

[3] J. R. Sharma, P. Gupta, An efficient fifth order method for solving system of nonlinear equations, Comp. Math. Appl., 67 (2014), 591-601.

[4] J. M. Ortega, W. C. Rheinboldt, Solution of nonlinear equation in several variables, Academic Press, New York, USA (1970).

[5] F. Ahmad, S. Hussain, N. A. Mir, A. Rafiq, New sixth order Jarrat method for solving nonlinear equations, Int. J. Appl. Math. Mech., 5 (5) (2009), 27-35.

[6] A. Cordero, J. R. Torregrosa, M. P. Vassileva, Increasing the order of convergence of iterative schemes for solving nonlinear system, J. Comp. App. Math., 252 (2013), 86-94.

[7] S. Amat, M. A. Hernandez, N. Romero, Semilocal convergence of a sixth order iterative method for quadratic equations, App. Numer. Math., 62 (2012), 833-841.

[8] V. Candela, A. Marquina, Recurrence relations for rational cubic method I: Halley method, Computing, 44 (2) (1990), 169-184.

[9] V. Candela, A. Marquina, Recurrence relations for rational cubic method II: Chebyshev method, Computing, 45 (4) (1990), 355-367.

[10] J. A. Ezqerro, M. A. Hernandez, Recurrence relations for Chebyshev-type methods, Appl. Math. Optim., 41 (2) (2000), 227-236.

[11] M. A. Hernandez, N. Romero, On a characterization of some Newton-like methods of R-order at least three, J. Comp. App. Math., 206 (2007), 873-887.

[12] X. Wang, J. Kou, G. Chuanqing, Semilocal convergence of a sixth-order Jarratt method in Banach spaces, Numer Algor, 57 (4) (2011), 441-456.

[13] L. Chen, C. Gu, Y. Ma, Semilocal covergence fifth-order Newton's method using recurrence relations in Banach spaces, J. Appl. Math. Article ID 786306 (2011), 15 pages.

[14] J. P. Jaiswal, Semilocal convergence of an eight-order method in Banach spaces and its computational efficiency, Numer Algor, 71 (4) (2015), 933-951.

[15] A. Cordero, M. A. Hernandez, N. Romero, J. R. Torregrosa, Semilocal convergence by using recurrence relations for a fifth-order method in Banach spaces, J. Comp. App. Math., 273 (2015), 205-213. 